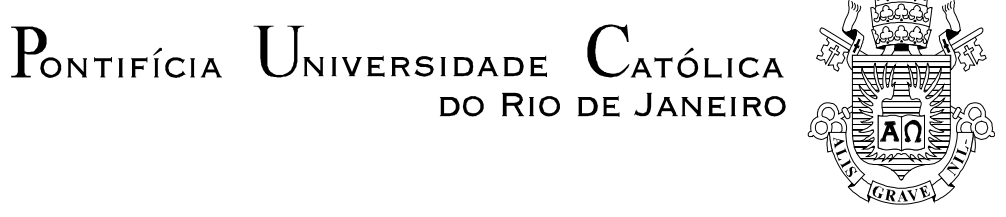

Oscar Cuaresma Zevallos

Perda e Inadequação do Controle de Tensão na Avaliação das Condições de Estabilidade de Tensão

Orientador: Prof. Ricardo Bernardo Prada 


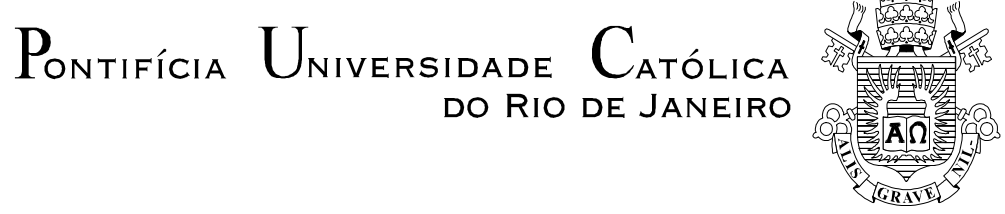

Oscar Cuaresma Zevallos

\section{Perda e Inadequação do Controle de Tensão na Avaliação das Condições de Estabilidade de Tensão}

Dissertação apresentada como requisito parcial para obtenção do grau de Mestre pelo Programa de Pós-Graduação em Engenharia Elétrica do Departamento de Engenharia Elétrica do Centro Técnico Científico da PUC-Rio. Aprovada pela Comissão Examinadora abaixo assinada.

Prof. Ricardo Bernardo Prada

Orientador Departamento de Engenharia Elétrica - PUC-Rio

Prof. Carlos Enrique Portugal Poma UFMT

Prof. Carlos Aparecido Ferreira Eletrobras - Centrais Elétricas Brasileiras

Prof. José Eugenio Leal Coordenador Setorial do Centro

Técnico Científico

Rio de Janeiro, 25 de abril de 2014 
Todos os direitos reservados. É proibida a reprodução total ou parcial do trabalho sem autorização da universidade, do autor e do orientador.

\section{Oscar Cuaresma Zevallos}

Graduado em Engenharia Elétrica pela Universidad Nacional de San Antonio Abad del Cusco (UNSAAC), em 2010 .

Ficha Catalográfica

Cuaresma Zevallos, Oscar

Perda e inadequação do controle de tensão na avaliação das condições de estabilidade de tensão / Oscar Cuaresma Zevallos; orientador: Ricardo Bernardo Prada. - Rio de Janeiro: PUC, Departamento de Engenharia Elétrica, 2014.

v., 146 f.: il. ; $30 \mathrm{~cm}$

Dissertação (mestrado)-Pontifícia Universidade Católica do Rio de Janeiro, Departamento de Engenharia Elétrica, 2014.

Inclui referências bibliográficas.

1. Engenharia elétrica - Teses. 2. Estabilidade de tensão. 3. Controle de tensão. 4. Adequação do controle de tensão. 5. Colapso de tensão. 6. Segurança de tensão. 7. Mudança no perfil de tensão. I. Prada, Ricardo Bernardo. II. Pontifícia Universidade Católica do Rio de Janeiro. Departamento de Engenharia Elétrica. III. Título.

CDD: 621.3 
A Nelly, minha mãe Por toda uma vida de infinito amor, compreensão e dedicação A Amarilis, minha irmã Por seu amor, compreensão e apoio incondicional nos momentos mais difíceis A Deus, por me mostrar o caminho certo nesta etapa da minha vida 


\section{Agradecimentos}

Ao Professor Ricardo Prada, pela dedicação, apoio incondicional e pela disposição para encaminhar as ideias e resolução das dúvidas durante desenvolvimento desta Dissertação.

À CAPES e à PUC-Rio, pelos auxílios financeiros concedidos.

Aos membros da Comissão da Pós-Graduação do DEE, pela oportunidade proporcionada de estudar meu Mestrado na PUC-Rio, no Brasil, e ais funcionários do Departamento de Engenharia Elétrica, por toda atenção prestada.

Ao Professor Delberis Lima, pelo incentivo prestado durante o estagio de docência.

À Javier Ortega, pelos bons conselhos e apoio constante.

A todos meus amigos do Laboratório de Sistemas de Energia, muito especialmente a Laura Paniagua, Erika Telles, Juan Vargas e Vanessa Gonzalez pelas alegrias prestadas no decorrer do curso.

À meu amigo e irmão Julio Córdova, pelo apoio incondicional e que esteve sempre torcendo pelo meu sucesso. 


\section{Resumo}

Zevallos, Oscar Cuaresma; Prada, Ricardo Bernardo. Perda e Inadequação do Controle de Tensão na Avaliação das Condições de Estabilidade de Tensão. Rio de Janeiro, 2014. 146p. Dissertação de Mestrado Departamento de Engenharia Elétrica, Pontifícia Universidade Católica do Rio de Janeiro.

Esta pesquisa está relacionada à avaliação das condições de estabilidade de tensão em pontos de operação do sistema elétrico. A modelagem de geradores / compensadores síncronos e compensadores estáticos são analisadas. Estabeleceram-se os melhores modelos com controle de tensão ativo e com perda do controle. A questão da adequação do controle de tensão foi tratada através da análise dos elementos da matriz de sensibilidade entre as grandezas controladoras e as tensões controladas. Os índices de estabilidade de tensão foram calculados. Em todos os casos, a perda de controle de tensão tem impacto negativo sobre as condições de estabilidade de tensão. Nos casos com ação de controle inadequada, independe da relação inversa entre grandeza controladora e tensão controlada, um aumento da tensão na barra controlada teve um impacto positivo sobre as condições de estabilidade de tensão, e uma diminuição da tensão na barra controlada teve impacto negativo sobre as condições de estabilidade de tensão. Esse é o resultado esperado também quando a ação de controle é adequada.

\section{Palavras - chave}

Estabilidade de tensão; controle de tensão; adequação do controle de tensão; colapso de tensão; segurança de tensão; mudança no perfil de tensão. 


\section{Abstract}

Zevallos, Oscar Cuaresma; Prada, Ricardo Bernardo (Advisor). Loss of Control and Inadequate Voltage Control Action in the Evaluation of Voltage Stability Conditions. Rio de Janeiro, 2014. 146p. MSc. Dissertation -Departamento de Engenharia Elétrica, Pontifícia Universidade Católica do Rio de Janeiro.

This research is related to the evaluation of voltage stability conditions of operating points of the power system. It has been studied the modelling of synchronous generators/synchronous compensators and static var compensators. The best models were established with active voltage control and with loss of control. The issue of voltage control was treated through analysis of the matrix sensitivity elements between the controlling variables and voltages at controlled buses. The voltage stability indexes were evaluated. In all cases, control loss proved to have a negative impact on voltage stability conditions. In cases with inadequate control action, results are non-dependent of the opposed relation between the controlling variable and voltage at controlling bus, an increase on voltage at controlled busbar proved to have a positive impact on voltage stability conditions, and a decrease on voltage at controlled busbar proved to have a negative impact on voltage stability conditions. This is also expected when the control action is appropriate.

\section{Keywords}

Voltage stability; voltage control; voltage control sensitivity; voltage collapse; voltage profile change. 


\section{Sumário}

1 Introdução 21

1.1. Considerações Gerais 21

1.2. Objetivos 22

1.3. Estrutura do Trabalho 22

2 Caracterização do Fenômeno e Avaliação das Condições de

Estabilidade de Tensão $\quad 24$

2.1. Introdução 24

2.2. Caracterização do Fenômeno de Estabilidade de Tensão 24

2.2.1. Curva $P$ e $Q$ Constante 27

2.2.2. Curva $\varnothing$ Constante 29

2.2.3. Impedância de Carga no Máximo Carregamento 30

2.3. Avaliação das Condições de Estabilidade de Tensão 35

2.3.1. Modulo da Determinante da Matriz D' 36

2.3.2. Sinal do Determinante da Matriz [D’] 39

2.3.3. Interpretação dos Índices 41

2.3.4. Aplicação em Sistema-Teste de 5 Barras 44

3 Modelagem de Geradores / Compensadores Síncronos e de Compensadores Estáticos após Perda do Controle de Tensão 47

3.1. Modelagem de Geradores / Compensadores Síncronos 47

3.1.1. Modelo Clássico do Gerador no Problema de Fluxo de Carga 47

3.1.2. Modelo Novo do Gerador no Problema de Fluxo de Carga 52

3.1.3. Perda de Controle de Tensão no Problema de Fluxo de Carga 61

3.1.3.1. Modelo Clássico do Gerador 62

3.1.3.2. Modelo Novo do Gerador 64

3.1.4. Análise de Estabilidade de Tensão Com Controle de Tensão 67

3.1.5. Análise de Estabilidade de Tensão Sem Controle de Tensão 68

3.1.6. Modelagem do Gerador Swing 68

3.1.7. Cálculo dos Índices no Sistema-Teste de 5 Barras 74 
3.1.7.1. Índices Com Controle Local de Tensão no Modelo Clássico e Novo Modelo do Gerador

3.1.7.2. Índices Sem Controle Local de Tensão no

Modelo Clássico e Novo Modelo do Gerador 78

3.1.7.3. Comparação dos Índices Com e Sem Controle de Tensão 83

3.2. Modelagem de Compensadores Estáticos (SVC) 85

3.2.1. Modelo do Compensador Estático no Problema de

Fluxo de Carga $\quad 88$

3.2.1.1. Regiões Capacitiva e Indutiva 89

3.2.1.2. Região Linear 91

3.2.1.3. Aplicação em Sistema-Teste de 14 Barras 92

3.2.2. Modelo da Susceptância Total e do Ângulo de Disparo do SVC no Problema de Fluxo de carga 95

3.2.2.1. Modelo da Susceptância Total do SVC 96

3.2.2.2. Modelo do Ângulo de Disparo do SVC 97

3.2.2.3. Controle do Módulo de Tensão por SVC 98

3.2.2.4. Aplicação em Sistema-Teste de 14 Barras 98

3.2.3. Sistema de Equações para o Cálculo dos Índices de

Estabilidade de Tensão $\quad 99$

3.2.3.1. Cálculo dos Índices de Estabilidade de Tensão sem Perda de Controle de Tensão 100

3.2.3.2. Cálculo dos Índices de Estabilidade de Tensão com Perda de Controle de Tensão

3.2.4. Comparação dos Resultados dos Índices de Estabilidade de Tensão Obtidos Sem e Com Perda de Controle de Tensão 102

4 Avaliação das Condições de Estabilidade de Tensão Após Atuação Inversa dos Equipamentos de Controle 103

4.1. Introdução 103

4.2. Matriz [VCS] dos Equipamentos de Controle 103

4.2.1. Análise da Matriz [VCS] 105

4.3. Índices de Estabilidade de Tensão em Pontos de Operação com Inadequação do Controle de Tensão 106

4.3.1. Sistema-Teste de 5 Barras 106 
4.3.1.1. Cálculo da Matriz [VCS] 106

4.3.1.2. Índices de Estabilidade de Tensão 111

4.3.2. Sistema-Teste de 14 Barras 112

4.3.2.1. Cálculo da Matriz [VCS] 112

4.3.2.2. Índices de Estabilidade de Tensão 118

4.4. Obtenção do Ponto de Operação Através da Matriz [VCS] 121

4.4.1. Sistema-Teste de 5 Barras 124

4.4.2. Sistema-Teste de 14 Barras 130

5 Conclusões e Trabalhos Futuros 142

5.1. Conclusões 142

5.2. Trabalhos Futuros 143

6 Referência Bibliográficas 145 


\section{Lista de figuras}

Figura 2.1. - Circuito de Duas Barras 26

Figura 2.2. - Curvas de V1 Constante no Plano $01 \mathrm{P} 1$

Figura 2.3. - Curvas PD1 e QD1 Constantes no Plano 01V1 27

Figura 2.4. - Curvas PD1 e QD1 Constantes no Plano 01 V1 com

Mesmo Fator de Potência 28

Figura 2.5. - Curvas Com $\phi$ Constante no Plano $\theta 1 \mathrm{~V} 1$

Figura 2.6. - Circuito de Duas Barras com Impedâncias de

Linha e de Carga

Figura 2.7. - Limite de Estabilidade de Tensão Sobre as Curvas de $\varnothing$

Constante no Plano S1V1

Figura 2.8. - Localização dos Vetores Gradiente $\nabla$ Pi e $\nabla Q i$ no Plano V $\theta$

Figura 2.9. - Curvas de $\varnothing$ Constante no Plano S1V1 43

Figura 2.10. - Sistema-Teste de 5 Barras 44

Figura 3.1. - Modelo clássico do Gerador com Controle Local 47

Figura 3.2. - Sistema-Teste de 5 Barras - Modelo Clássico com Controle Local 48

Figura 3.3. - Modelo Clássico do Gerador com Controle Remoto 51

Figura 3.4. - Sistema-Teste de 5 Barras - Modelo Clássico com Controle Remoto 51

Figura 3.5. - Modelo Novo do Gerador com Controle Local 53

Figura 3.6. - Sistema de 5 Barras - Modelo Novo do Gerador com Controle Local $\quad 55$

Figura 3.7. - Modelo Novo do Gerador com Controle Remoto $\quad 57$

Figura 3.8. - Sistema de 5 Barras - Modelo Novo do Gerador com Controle Remoto 59

Figura 3.9. - Ponto de Operação Estável com Limite Atingido 62

Figura 3.10.- Ponto de Operação Instável com Limite Atingido 63

Figura 3.11. - Curva de Atuação do Regulador Automático de Tensão 68 
Figura 3.12. - Sistema de 2 Barras - Modelo Clássico do

Gerador Swing 69

Figura 3.13. - Sistema de 2 Barras - Modelo Novo do Gerador Swing 70

Figura 3.14. - Estrutura do SVC 85

Figura 3.15. - Impedância Equivalente do SVC em Função do

Ângulo de Disparo 86

Figura 3.16. - Susceptância Equivalente do SVC em Função do Ângulo de Disparo $\quad 87$

Figura 3.17. - Regiões de Operação do Equipamento (SVC) 90

Figura 3.18. - Sistema-Teste de 14 Barras 92

Figura 3.19. - Modelo do SVC como Susceptância Shunt Variável 96

Figura 4.1. - Sistema-Teste de 14 Barras 113

Figura 4.2. - Relação Usual entre a Grandeza Controladora e a Tensão Controlada

Figura 4.3. - Relação Não Usual entre a Grandeza Controladora e a Tensão Controlada

Figura 4.4. - Relação com Transição de Região de Operação Entre a Grandeza Controladora e a Tensão Controlada

Figura 4.5. - Sistema-Teste de 5 Barras

Figura 4.6. - Relação Entre a Tensão Interna e a Tensão

Terminal do Gerador 1

Figura 4.7. - Relação Entre a Tensão Interna e a Tensão

Terminal do Gerador 2

Figura 4.8. - Relação entre a Tensão Interna e a Tensão

Terminal do Gerador 1

Figura 4.9. - Relação entre a Tensão Interna e a Tensão

Terminal do Gerador 2

Figura 4.10. - Relação entre a Tensão Interna e a Tensão

Terminal do Gerador 3

Figura 4.11. - Relação entre a Tensão Interna e a Tensão

Terminal do Gerador 4

Figura 4.12. - Relação entre a Tensão Interna e a Tensão

Terminal do Gerador 5 
Figura 4.13. - Relação entre o Ângulo de Disparo e a Tensão

Controlada da Barra 14 


\section{Lista de tabelas}

Tabela 2.1. - Dados do Circuito de Duas Barras 25

Tabela 2.2. - Dados de Barra do Sistema-Teste de 5 Barras 44

Tabela 2.3. - Dados de Linha do Sistema-Teste de 5 Barras 44

Tabela 2.4. - Resultado do Fluxo de Carga do Sistema-Teste de

5 Barras

Tabela 2.5. - Índices de Estabilidade de Tensão do Sistema-Teste de

5 Barras

Tabela 3.1. - Dados de Barra do Sistema de 5 Barras - Controle Local 48

Tabela 3.2. - Dados de Linha do Sistema de 5 Barras - Controle Local 48

Tabela 3.3. - Resultado do Fluxo de Carga do Sistema de 5 Barras -

Controle Local

Tabela 3.4. - Dados de Barra do Sistema de 5 Barras - Controle

Remoto

Tabela 3.5. - Dados de Linha do Sistema de 5 Barras - Controle

Remoto

Tabela 3.6. - Resultado do Fluxo de Carga do Sistema de 5 Barras

Controle Remoto

Tabela 3.7. - Dados do Sistema de 5 Barras - Modelo Novo com

Controle Local

Tabela 3.8. - Dados de Linha do Sistema de 5 Barras

Modelo Novo com Controle Local

Tabela 3.9. - Resultado do Fluxo de Carga do Sistema de 5 Barras Modelo Novo com Controle Local

Tabela 3.10. - Dados do Sistema de 5 Barras - Modelo Novo com

Controle Remoto

Tabela 3.11. - Dados de Linha do Sistema de 5 Barras - Modelo

Novo com Controle Remoto

Tabela 3.12. - Resultado do Fluxo de Carga do Sistema de 5 Barras

Modelo Novo com Controle Remoto

Tabela 3.13. - Dados de barra e de linha do sistema de 2 barras 
Tabela 3.14. - Dados de Barra e de Linha do Sistema de 2 Barras Modelo Novo do Gerador Swing

Tabela 3.15. - Resultados de Fluxo de Carga e dos Índices da Barra 2 no Modelo Clássico e Novo Modelo do Gerador - Caso 170

Tabela 3.16. - Resultados de Fluxo de Carga e dos Índices da Barra 2 no Modelo Clássico e Novo Modelo do Gerador - Caso 2

Tabela 3.17. - Resultados de Fluxo de Carga e dos Índices da Barra 2 no Modelo Clássico e Novo Modelo do Gerador - Caso 3

Tabela 3.18. - Resultados de Fluxo de Carga e dos Índices da Barra 2 no Modelo Clássico e Novo Modelo do Gerador - Caso 4

Tabela 3.19. - Matriz D e Matriz D' da Barra 2 com o Modelo Clássico no Gerador Swing

Tabela 3.20. - Sub-Matrizes D, C, A, B e Matriz D' da Barra 2 com o Novo Modelo no Gerador Swing

Tabela 3.21. - Matriz D e Matriz D' da Barra 2 com o Modelo Clássico no Gerador Swing

Tabela 3.22. - Sub-Matrizes D, C, A, B e Matriz D' da Barra 2 com o Novo Modelo no Gerador Swing

Tabela 3.23. - Índices de Estabilidade de Tensão do Sistema de 5 Barras Modelo Clássico

Tabela 3.24. - Índices de Estabilidade de Tensão do Sistema de 5 Barras Modelo Novo com Controle de Tensão de Excitação

Tabela 3.25. - Índices de Estabilidade de Tensão do Sistema de 5 Barras Modelo Novo com Controle de Potência Reativa Gerada

Tabela 3.26. - Sub-Matrizes D, C, A, B e Matriz D' da Barra 3 no Modelo Clássico

Tabela 3.27. - Sub-Matrizes D, C, A, B e Matriz D' da Barra 3 no Modelo Novo

Tabela 3.28. - Índices de Estabilidade de Tensão do Sistema de 5 Barras Modelo Clássico com Perda de Controle

Tabela 3.29. - Índices de Estabilidade de Tensão do Sistema de 5

Barras Modelo Novo com Perda de Controle

Tabela 3.30. - Índices de Estabilidade de Tensão do Sistema de 5

Barras Modelo Novo com Perda de Controle 
Tabela 3.31. - Sub-Matrizes D, C, A, B e Matriz D' da Barra 3 no Modelo Clássico

Tabela 3.32. - Sub-Matrizes D, C, A, B e Matriz D' da Barra 3 no Modelo Novo sem Controle de Tensão pela Tensão de Excitação

Tabela 3.33. - Sub-Matrizes D, C, A, B e Matriz D' da Barra 3 no Modelo Novo sem Controle de Tensão pela Potência Reativa Gerada 81

Tabela 3.34. - Dados de Barra do Sistema de 14 Barras 93

Tabela 3.35. - Dados de Linha do Sistema de 14 Barras 93

Tabela 3.36. - Dados do SVC do Sistema de 14 Barras 93

Tabela 3.37. - Resultado de Fluxo de Carga do Sistema de 14 Barras 94

Tabela 3.38. - Tensões Máximas e Mínimas do SVC 94

Tabela 3.39. - Resultados do Ângulo, Susceptância e Potência Reativa Gerada do SVC Após Convergência 95

Tabela 3.40. - Resultado de Fluxo de Carga do Sistema de 14 Barras 98

Tabela 3.41. - Resultados da Susceptância Equivalente nos

Dois Modelos

Tabela 3.42. - Índices do Sistema de 14 Barras Sem Perda de

Controle de Tensão

Tabela 3.43. - Dados do Equipamento (SVC) do Sistema de 14 Barras

Tabela 3.44. - Índices do Sistema de 14 Barras

Com Perda de Controle

Tabela 4.1. - Ponto de Operação e Dados de Linha do

Sistema-Teste de 5 Barras

Tabela 4.2. - Variação de Tensão na Barra Interna Após Aumento em 0,01 p.u. nas Barras Terminais dos Geradores 1 e 2

Tabela 4.3. - Ponto de Operação e Dados de Linha do Sistema-Teste de 5 Barras

Tabela 4.4. - Variação de Tensão na Barra Interna Após Aumento em 0,01 p.u. nas Barras Terminais dos Geradores 1 e 2

Tabela 4.5. - Índices de Estabilidade de Tensão do Sistema-Teste de 5 Barras - Caso-Base 
Tabela 4.6. - Índices de Estabilidade de Tensão do Sistema-Teste de 5 Barras - Após Aumento em 0,01 p.u. nas Barras Terminais dos Geradores

Tabela 4.7. - Dados de Reatância dos Geradores/Compensadores Síncronos do Sistema-Teste de 14 Barras

Tabela 4.8. - Resultado de Fluxo de Carga do Sistema-Teste de 14 Barras

Tabela 4.9. - Variação de Tensão da Barra Interna e do Ângulo de

Disparo Após Diminuição de Tensão nas Barras Controladas 8 e $14 \quad 115$

Tabela 4.10. - Variação de Tensão da Barra Interna e Ângulo de

Disparo Após Diminuição de Tensão na Barra 8 e Incremento de

Tensão na Barra 14

Tabela 4.11. - Variação de Tensão da Barra Interna Após

Diminuição de Tensão na Barra Controlada 8

Tabela 4.12. - Variação de Tensão da Barra Interna Após

Incremento de Tensão na Barra Controlada 14

Tabela 4.13. - Variações das Tensões Internas e do Ângulo de

Disparo à Ação Conjunta das Ações de Controle

Tabela 4.14. - Índices de Estabilidade de Tensão do Sistema-Teste de 14 Barras - Caso-Base

Tabela 4.15. - Índices de Estabilidade de Tensão do Sistema-Teste de 14 Barras Após Diminuição de Tensão nas Barras

Controladas 8 e 14

Tabela 4.16. - Índices de Estabilidade de Tensão do Sistema-Teste de 14 Barras Após Diminuição de Tensão da Barra Controlada 8 e Aumento de Tensão da Barra Controlada 14

Tabela 4.17. - Dados de Barra do Sistema de 5 Barras

Tabela 4.18. - Dados de Linha do Sistema de 5 Barras

Tabela 4.19. - Resultado do Fluxo de Carga do Sistema de 5 Barras Caso-Base

Tabela 4.20. - Mudança do Perfil de Tensão nas barras 1 e 2 do Sistema-Teste de 5 Barras

Tabela 4.21. - Matriz [VCS] e Variação da Tensão Interna para Cada Passo 
Tabela 4.22. - Resultado do Fluxo de Carga Após Mudança do Perfil de Tensão $\quad 129$

Tabela 4.23. - Índices de Estabilidade de Tensão Caso-Base $\quad 129$

Tabela 4.24. - Índices de Estabilidade de Tensão no Novo Ponto de Operação

Tabela 4.25. - Mudança do Perfil de Tensão nas barras 8 e 14 do Sistema-Teste de 14 Barras

Tabela 4.26. - Limites Operacionais nas Barras Internas 18 e 19 do

Sistema-Teste de 14 Barras

Tabela 4.27. - Resultado de Fluxo de Carga do Novo Perfil de Tensão do Sistema-Teste de 14 Barras

Tabela 4.28. - Índices de Estabilidade de Tensão do Sistema-Teste de 14 Barras - Caso-Base

Tabela 4.29. - Índices de Estabilidade de Tensão Após Mudança do Perfil de Tensão 


\section{Lista de Abreviaturas e Siglas}

\begin{tabular}{|c|c|}
\hline CS & Compensador Síncrono \\
\hline II & Índice de Influência \\
\hline IEEE & Institute of Electrical and Electronics Engineers \\
\hline LTC & Load Tap Changing \\
\hline LEEA & Limite de Estabilidade Estática Angular \\
\hline LET & Limite de Estabilidade de Tensão \\
\hline RAT & Regulador Automático de Tensão \\
\hline SVC & Static Var Compensator \\
\hline TCR & Thyristor Controlled Reactor \\
\hline [VCS] & Matriz de Sensibilidade dos Equipamentos de Controle \\
\hline M & Margem de Potência \\
\hline$M_{o}$ & Margem de Potência no Caso-Base \\
\hline$M_{1}$ & Margem de Potência Após o Evento em Análise \\
\hline$P$ & Potência Ativa \\
\hline Q & Potência Reativa \\
\hline$\theta$ & Ângulo da Tensão \\
\hline $\mathrm{V}$ & Módulo da Tensão \\
\hline$E_{g}$ & Módulo da Tensão Interna do Gerador \\
\hline$\Delta P$ & Resíduo da Potência Ativa \\
\hline$\Delta Q$ & Resíduo da Potência Reativa \\
\hline$\Delta \theta$ & Variação Incremental do Ângulo da Tensão \\
\hline$\Delta V$ & Variação Incremental do Módulo da Tensão \\
\hline \multirow[t]{2}{*}{$\Delta E_{g}$} & Variação Incremental do Módulo da Tensão Interna do \\
\hline & Gerador \\
\hline$[\mathrm{J}]$ & Matriz Jacobiana \\
\hline$[A]$ & Submatriz da matriz Jacobiana expandida \\
\hline [B] & Submatriz da matriz Jacobiana expandida \\
\hline$[\mathrm{C}]$ & Submatriz da matriz Jacobiana expandida \\
\hline [D] & Submatriz da matriz Jacobiana expandida \\
\hline [D’] & $\begin{array}{l}\text { Matriz obtida de uma redução da matriz Jacobiana } \\
\text { expandida }\end{array}$ \\
\hline
\end{tabular}




$\begin{array}{ll}S_{i} & \text { Injeção de Potência Aparente na Barra i } \\ S_{m} & \text { Injeção Máxima de Potência Aparente na Barra i } \\ \nabla P & \text { Gradiente do Fluxo de Potência Ativa } \\ \nabla Q & \text { Gradiente do Fluxo de Potência Reativa } \\ \beta & \text { Ângulo entre os Gradientes dos Fluxos de Potência Ativa e } \\ & \text { Reativa } \\ Z & \text { Módulo da Impedância } \\ \varnothing & \text { Fator de Potência na Carga } \\ \hat{I}_{01} & \text { Fasor da Corrente de Saída do Gerador } \\ \hat{V}_{o} & \text { Fasor de Tensão em Terminais do Gerador } \\ \hat{Z}_{t} & \text { Fasor da Impedância da Linha de Transmissão } \\ \hat{Z}_{c} & \text { Fasor da Impedância da Carga } \\ \varepsilon & \text { Banda Morta do Compensador Estático } \\ \alpha & \text { Ângulo de Disparo do Compensador Estático } \\ B_{e q} & \text { Susceptância Equivalente do Compensador Estático } \\ X_{C} & \text { Reatância Capacitiva do Compensador Estático } \\ X_{L} & \text { Reatância Indutiva do Compensador Estático } \\ X_{s l} & \text { Inclinação da Reta de Controle do Compensador Estático } \\ X_{s} & \text { Reatância Síncrona em Régime Permanente }\end{array}$

